\title{
FLORA VASCULAR DE AÇUDES DE UMA REGIÃO DO SEMI-ÁRIDO DA BAHIA, BRASIL ${ }^{1}$
}

\author{
Flávio França ${ }^{2}$ \\ Efigênia de Melo $^{2}$ \\ Aristóteles Góes Neto² \\ Dione Araújo ${ }^{3}$ \\ Márcia G. Bezerra² \\ Helayra M. Ramos ${ }^{2}$ \\ Ivan Castro ${ }^{2}$ \\ Doriedson Gomes ${ }^{2}$
}

Recebido em 03/10/1999. Aceito em 10/05/2003

\begin{abstract}
RESUMO - (Flora vascular de açudes de uma região do semi-árido da Bahia, Brasil). Foram selecionados seis açudes que margeiam a Estrada do Feijão (BA 052), nos municípios de Feira de Santana e Angüera, entre as coordenadas $39^{\circ} 30^{\prime}-39^{\circ} 00^{\prime} \mathrm{W}$ e $12^{\circ} 00^{\prime}-12^{\circ} 30^{\prime} \mathrm{S}$. Com a finalidade de se conhecer a flora vascular dos mesmos, esses tipos de plantas foram coletadas de agosto/1996 a dezembro/1997. Registraram-se 121 espécies distribuídas em 46 famílias. As famílias mais representativas foram: Cyperaceae, com 18 espécies (14,9\%), Poaceae, com 13 (10,7\%), Asteraceae, com 10 (8,3\%), Scrophulariaceae e Fabaceae, com 6 (5,0\%). As espécies que ocorreram em todos os açudes foram Pistia stratiotes L. (Araceae), Echinochloa colona (L.) Link (Poaceae), Nymphaea ampla (Salisb.) DC. (Nymphaeaceae) e Oxycarium cubense (Poepp. \& Kunth.) Lye (Cyperaceae).
\end{abstract}

Palavras-chave - plantas aquática, semi-árido, Bahia, açude

\begin{abstract}
Pond vascular flora in the semi-arid region of Bahia State, Brazil). Six ponds were studied between August/1996 and December/1997 at the edge of State Highway BA 052, in the municipalities of Feira de Santana and Angüera, $39^{\circ} 30^{\prime}-39^{\circ} 00^{\prime} \mathrm{W}$ and $12^{\circ} 00^{\prime}-12^{\circ} 30^{\prime} \mathrm{S}$. With the aim to know their vascular flora, one hundred and twenty-one species of vascular plants were collected, representing 46 families. The most frequent families were: Cyperaceae with 18 species (14.9\%), Poaceae with 13 (10.7\%), Asteraceae with 10 (8.3\%), Scrophulariaceae and Fabaceae with $6(5.0 \%)$. The species which were recorded in all lakes were: Pistia stratiotes L. (Araceae), Echinochloa colona (L.) Link (Poaceae), Nymphaea ampla (Salisb.) DC. (Nymphaeaceae) and Oxycarium cubense (Poepp. \& Kunth.) Lye (Cyperaceae).
\end{abstract}

Key words - aquatic plants, semi-arid, Bahia, pond

1 Projeto Institucional da UEFS, apoio emergencial do CNPq (Processo 42.0045/96)

2 Departamento de Ciências Biológicas (DCBIO), Universidade Estadual de Feira de Santana (UEFS), km 3, BR-116 N, Campus Universitário, CEP 44031-460, Feira de Santana, BA, Brasil (flaviofranca@ hotmail.com)

3 Departamento de Tecnologia, Laboratório de Saneamento, Bioquímica, Universidade Estadual de Feira de Santana, UEFS 


\section{Introdução}

A vegetação de ambientes aquáticos no Estado da Bahia é pouco conhecida, particularmente de ambientes lênticos do semi-árido. Na região de Feira de Santana e Angüera, devido aos rigores dos longos períodos de estiagem, tornou-se tradicional o represamento de pequenos cursos de água, geralmente temporários. Os açudes originados destes represamentos acabaram sustentando flora vascular bastante diversa e pouco estudada.

O conhecimento da vegetação aquática é relevante para a melhor racionalização do uso dos recursos hídricos na região, fornecendo alternativas para despoluição e alimentação de diversas criações animais (Albuquerque 1981), além de algumas espécies servirem como indicadoras de poluição (Pedralli 1996). Deve-se ressaltar ainda que o conhecimento científico dos processos físicos, químicos e biológicos destes corpos aquáticos no semi-árido é deficiente (Tundisi 1994).

Este trabalho teve como objetivo conhecer a composição florística vascular da área em estudo, assim como avaliar a sua relação com alguns parâmetros físico-químicos.

\section{Material e métodos}

Foram selecionados açudes formados pelo represamento de cursos d'água temporários que estivessem com alta cobertura de macrófitas. $\mathrm{Na}$ margem de cada açude, um marco de madeira foi colocado com o objetivo de medir a amplitude do nível da água.

As amostras de plantas vasculares férteis e de água para análise química foram coletadas mensalmente em cada açude, no período de agosto/1996 a dezembro/1997.

Em três ocasiões diferentes foram realizadas coletas de água ao longo de 24 horas. O Açude 5 foi o escolhido para estas coletas devido a conveniências logísticas. As amostras de água foram colhidas às 17:30, 23:30, 5:30, 11:30 e novamente às 17:30h.
A coleta de plantas vasculares foi realizada segundo a metodologia de Fidalgo \& Bononi (1995), sem delineamento estatístico.

No caso da massa flutuante, formada por fanerógamas e pteridófitas diminutas, uma amostra foi colhida mensalmente e triada no laboratório. Uma vez separadas, as morfoespécies foram individualizadas em frascos e imersas em álcool 70\%. Parte das amostras foi separada para montagem de exsicatas.

O sistema de classificação adotado para as fanerógamas foi o de Cronquist (1981) e, para as pteridófitas, o de Tryon \& Tryon (1982).

A identificação ao nível de espécie foi realizada por especialistas; na ausência dos mesmos, o material foi identificado com base na coleção do Herbário HUEFS e com o auxílio de literatura especializada.

A classificação dos tipos de formas biológicas das espécies baseou-se em Irgang et al. (1984).

O cálculo do índice de similaridade de Sørensen (S) segue Mueller-Dombois \& Ellenberg (1974) e Felfili et al. (1994). Os índices obtidos foram objetos de análises de agrupamentos utilizando-se NTSYS-PC 1.8, através dos programas UPGMA, método de ligamento por média aritmética não ponderada; COMPLETE, método de ligamento completo e SINGLE, método de ligamento simples. Os dendrogramas originados foram expostos na forma de notação parentética, acrescentando-se os valores do nível de similaridade de cada grupo formado (Crisci \& Armengol 1983; Rohlf 1997).

Os parâmetros físico-químicos da água avaliados foram: fosfato total, salinidade e alcalinidade, temperatura (medida in situ) e $\mathrm{pH}$ (medido no laboratório). As amostras de água para a análise foram coletadas na margem do açude em frascos plásticos com capacidade de um litro, transportadas à temperatura ambiente até o laboratório, onde eram mantidas à temperatura de $5^{\circ} \mathrm{C}$ até o dia seguinte quando eram analisadas. 
Os métodos para determinação dos parâmetros químicos estão em Clesceri et al. (1989). Para a determinação de fósforo total utilizou-se o método do ácido ascórbico. A alcalinidade foi medida através do método da titulação. Para a salinidade, utilizou-se o método argentométrico.

Os dados climatológicos foram obtidos na estação meteorológica instalada no Campus da UEFS, ca. 20km NE do açude 1 . O diagrama pluviométrico foi elaborado conforme aqueles apresentados em Nimer (1989)

A área de estudos (Fig. 1) está localizada ao longo da BA 052 (Estrada do Feijão), no trecho entre o $\mathrm{km} 0$ e o $\mathrm{km} 30$, entre as coordenadas $39^{\circ} 30^{\prime}-39^{\circ} 00^{\prime} \mathrm{W}$ e $12^{\circ} 00^{\prime}-12^{\circ} 30^{\prime} \mathrm{S}$, pertencente aos municípios de Feira de Santana e Angüera, ambos incluídos entre os municípios da região semi-árida baiana (Bahia 1995).

Foram selecionados 6 açudes:

Açude 1 - localizado no município de Feira de Santana, nas coordenadas $12^{\circ} 16^{\prime} 24^{\prime \prime} \mathrm{S}$ e $39^{\circ} 03^{\prime} 10^{\prime}$ 'W, km 3 da BA 052, $160 \mathrm{~m}$ de altitude, com ca. $270 \mathrm{~m}^{2}$ de área.

Açude 2 - localizado no município de Feira de Santana, nas coordenadas $12^{\circ} 16^{\prime} 32^{\prime \prime S ~ e ~}$ 39 03 '22' $\mathrm{W}, \mathrm{km} 3$ da BA 052, 160m de altitude, com ca. $4.270 \mathrm{~m}^{2}$. Os açudes 1 e 2 são separados pela estrada e provavelmente há comunicação entre eles.

Açude 3 - localizado no município de Angüera, nas coordenadas $12^{\circ} 13^{\prime} 30^{\prime} \mathrm{S}$ e 39 $06^{\prime} 42^{\prime \prime} \mathrm{W}, \mathrm{km} 11$ da BA 052, 170m de altitude, com ca. $2.440 \mathrm{~m}^{2}$. Uma característica deste açude é o seu leito rochoso de origem gnáissica.

Açude 4 - localizado no município de Angüera, nas coordenadas $12^{\circ} 13$ ' $24^{\prime \prime} \mathrm{S}$ e 39 06'59'W, km 12 da BA 052, 170m de altitude, com ca. $2.300 \mathrm{~m}^{2}$.

Açude 5 - localizado no município de Angüera, nas coordenadas $12^{\circ} 11$ ' $38^{\prime}$ S e 39 $09^{\circ} 07^{\prime \prime} \mathrm{W}, \mathrm{km} 16$ da BA 052, 200m de altitude, com ca. $5.860 \mathrm{~m}^{2}$. Os moradores locais afirmam que este açude é muito antigo, tendo sido "escavado pelos escravos".
Açude 6 - localizado no município de Angüera, nas coordenadas $12^{\circ} 10^{\prime} 00^{\prime \prime} \mathrm{S}$ e 39 $12^{\prime} 36^{\prime}$ 'W, $\mathrm{km} 23$ da BA 052, 200m de altitude, com ca. $12.900 \mathrm{~m}^{2}$.

\section{Resultados}

A temperatura da água dos açudes, assim como o pH, não variaram muito durante a realização dos trabalhos, sendo que os açudes apresentaram leve acidez, em média.

Os menores valores de alcalinidade foram encontrados no açude 3 e os maiores, no açude 1. $\mathrm{O}$ açude 6 apresentou os maiores valores de fósforo total, enquanto os menores foram observados no açude 2 . $\mathrm{O}$ açude 4 foi o que apresentou água mais salina e o açude 2 mostrou-se menos salino. Todos esses valores mostraram grande variação ao longo do ano. Os valores médios dos parâmetros físico-químicos analisados estão resumidos na Tabela 1 .

Quanto à variação da amplitude do nível da água, observou-se maior retração entre janeiro e fevereiro e a maior ampliação, em maio, estabelecendo uma área de depleção média de $8 \mathrm{~m}$.

A umidade relativa do ar variou de 75 a $92 \%$, sendo menor entre setembro e dezembro/1997 e maior entre maio e junho/1997. A temperatura atmosférica manteve-se razoavelmente constante, variando de $22,6^{\circ} \mathrm{C}$ a $28,6^{\circ} \mathrm{C}$, sendo que o mês mais quente foi janeiro/1997 e o mais frio, agosto/1997. A precipitação variou de 6,5 a $352,8 \mathrm{~mm}$, com dois períodos de pico em novembro/1996 e em março/1997.

O diagrama pluviométrico (Fig. 2) mostra que o período estudado apresentou sete meses secos, representando 58,3\% dos meses.

No acompanhamento dos valores dos parâmetros fisico-químicos ao longo de 24 horas (Tab. 2), a temperatura da água mostrou-se muito fria na noite e na madrugada da coleta realizada em agosto/1997, no início do período seco; os demais valores não variaram significativamente em relação àqueles coletados mensalmente. $\mathrm{O}$ pH foi significativamente mais ácido 
Tabela 1. Valores médios $(\times)$ dos parâmetros físico-químicos da água dos açudes. Temperatura (T), Potencial Hidrogeniônico (pH), Alcalinidade (A), Salinidade (Sal) e Fosfato total (Ptot), com os respectivos coeficientes de variação (cv).

\begin{tabular}{|c|c|c|c|c|c|c|c|c|c|c|}
\hline \multirow[t]{2}{*}{ Açude } & \multicolumn{2}{|c|}{$\mathrm{T}$} & \multicolumn{2}{|c|}{$\mathrm{pH}$} & \multicolumn{2}{|c|}{ A } & \multicolumn{2}{|c|}{ Sal } & \multicolumn{2}{|c|}{ Ptot } \\
\hline & $\times{ }^{\circ} \mathrm{C}$ & $\mathrm{cv} \%$ & $x$ & $\mathrm{cv} \%$ & $\times \mathrm{mEq} / \mathrm{l}$ & $\mathrm{cv} \%$ & $\overline{\times \mathrm{mg} / \mathrm{l}}$ & $\mathrm{cv} \%$ & $\overline{\times \mu \mathrm{g} / \mathrm{l}}$ & $\mathrm{cv} \%$ \\
\hline 1 & 24,80 & 10,20 & 6,97 & 10,8 & 2,57 & 57,6 & 23,0 & 57,7 & 119 & 69 \\
\hline 2 & 25,85 & 9,40 & 6,39 & 11,1 & 0,58 & 40,2 & 12,4 & 63,1 & 85 & 95 \\
\hline 3 & 25,97 & 12,10 & 6,39 & 10,6 & 0,60 & 67,5 & 51,8 & 45,8 & 102 & 82 \\
\hline 4 & 25,10 & 9,50 & 6,46 & 9,1 & 0,84 & 48,4 & 174,4 & 49,4 & 130 & 77 \\
\hline 5 & 25,05 & 11,60 & 6,83 & 17,1 & 0,88 & 51,8 & 49,6 & 37,9 & 116 & 43 \\
\hline 6 & 26,00 & 9,42 & 6,83 & 12,3 & 0,61 & 38,7 & 18,0 & 21,6 & 159 & 72 \\
\hline
\end{tabular}

das $5: 30$ às $17: 30 \mathrm{~h}$ na coleta realizada em abril/1997, um dos meses mais úmidos. A alcalinidade foi significativamente mais baixa na coleta de abril/1997. Os valores de salinidade foram significativamente mais baixos na coleta de abril/1997 e mais altos na coleta de dezembro/1997, respectivamente período úmido e período seco. Os valores de fosfato total foram maiores durante quase toda a coleta de dezembro/1997.
Foram coletadas 121 espécies distribuídas em 46 famílias (Tab. 3). O açude 5 foi o que apresentou o maior número de espécies com 51 $(42,2 \%)$, seguido pelo açude 6 , com 48 (39,7\%), açude 2 , com $46(38,0 \%)$, açude 3 , com 40 $(33,1 \%)$, açude 1 , com $32(27,8 \%)$ e açude 4 , com $29(24,0 \%)$.

Cyperaceae, com 18 espécies $(14,9 \%)$ e Poaceae, com 13 espécies $(10,7 \%)$, foram as famílias melhor representadas, seguidas por
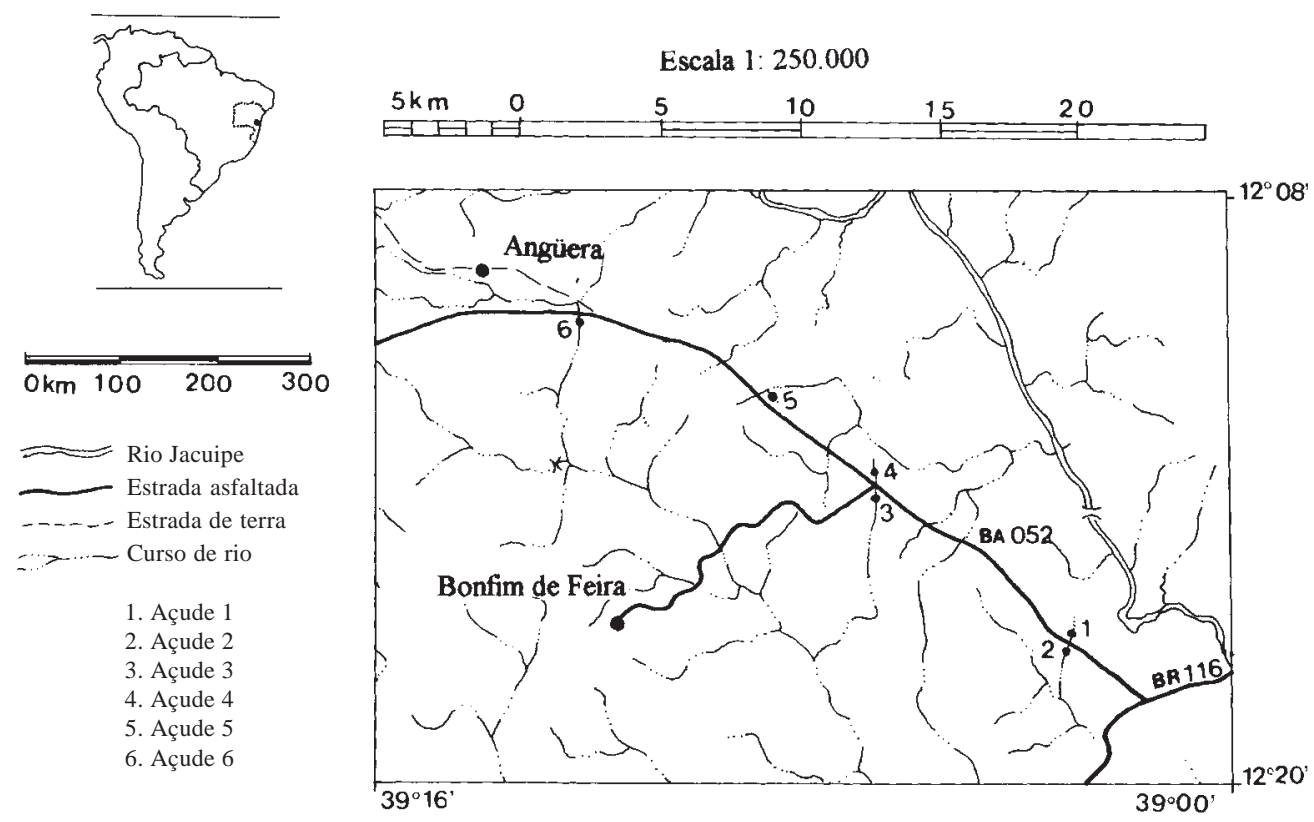

Figura 1. Mapa da área de estudo (Fonte: Brasil s.d. Folha Topográfica SD 24-V-B, 1:250.000). $1=$ Açude 1; 2 = Açude 2;3 = Açude 3; $4=$ Açude 4; $5=$ Açude 5; $6=$ Açude 6 . 
Asteraceae, com 10 espécies (8,3\%), Scrophulariaceae e Fabaceae, com 6 espécies (5,0\%).

As espécies com maior distribuição, ou seja, presentes em pelo menos cinco açudes foram: Pistia stratiotes* (Araceae), Cyperus surinamensis e Oxycarium cubense* (Cyperaceae), Aeschynomene evenia (Fabaceae), Hydrolea espinosa (Hydrophylaceae),

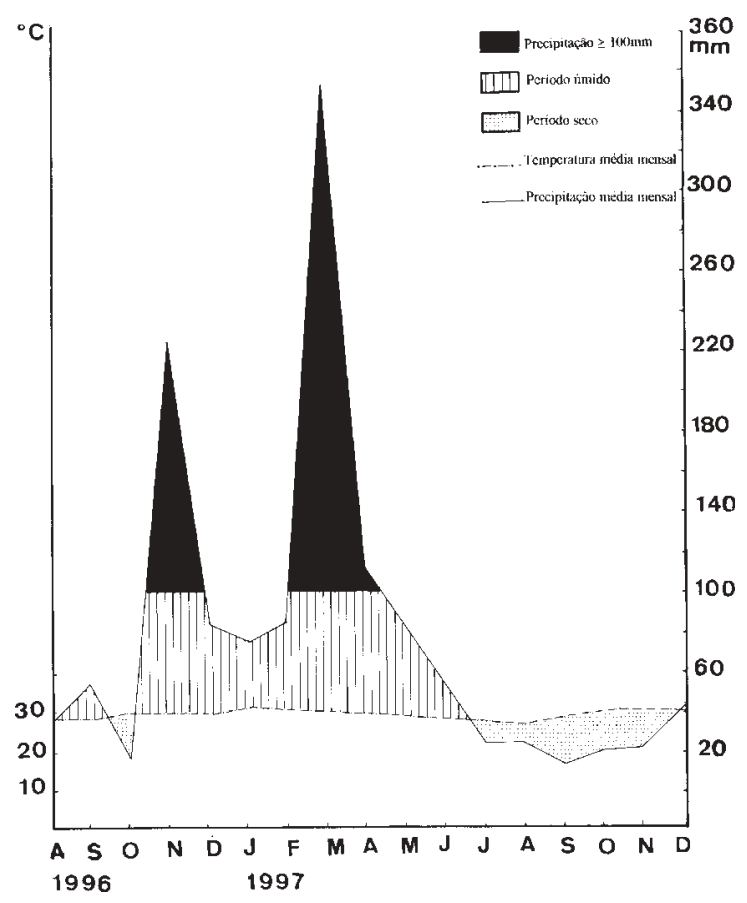

Figura 2. Diagrama pluviométrico (agosto/1996dezembro/1997). Estação meteorológica da Universidade Estadual de Feira de Santana (alt. 210m, lat. 12¹2’S).
Wolffiella welwitschii e Lemna cf. valdiviana (Lemnaceae), Utricularia gibba (Lentibulariaceae), Pleurophora anomala (Lythraceae), Nymphaea ampla* (Nymphaeaceae) e Echinocloa colona* (Poaceae), sendo as marcadas com asterisco aquelas observadas em todos os açudes.

Cada açude apresentou, em média, cerca de 11 espécies exclusivas. $O$ açude 5 apresentou o maior número de espécies exclusivas (16 spp.) e o 4 o menor número (cinco spp.).

Considerando o número de espécies compartilhadas por dois açudes, observa-se que o par de açudes 2-5 tem o maior número de espécies em comum (22 spp.) e o par de açudes 1-4 tem o menor número (12 spp.). Cada par de açude, no total de 15 pares possíveis, compartilha 16 espécies em média.

Usando o índice de similaridade de Sørensen (S) para os 15 pares de açudes possíveis, observou-se que $\mathrm{S}$ foi sempre menor que 0,5 . Os pares com $\mathrm{S}$ maior que 0,45 foram $4-6$ $(\mathrm{S}=0,4675)$ e $2-5(\mathrm{~S}=0,4536)$. Aqueles com $\mathrm{S}$ menor que 0,35 foram 2-3 $(0,3488)$ e $2-4$ $(0,3466)$. O valor médio de $\mathrm{S}$ ficou em 0,4087 (coeficiente de variação $8,80 \%$ ).

As análises de agrupamento resultaram em três dendrogramas, diferentes: 1- UPGMA: (3, ((2,5): 0.454, (1, (4,6): 0.468): 0.426): 0.404): 0.388; 2- SINGLE: $((4,6): 0.468,(1,(3,(2,5): 0.454)$ : 0.440): 0.434): 0.428; 3- COMPLETE: $((2,5)$ : $0.454,(3,(1,(4,6): 0.468): 0.425): 0.361): 0.347$.

Tabela 2. Valores absolutos dos parâmetros físico-químicos em 24 horas, em três épocas diferentes: I- 05-06/abr/1997; II- 23-24/ago/1997; III- 13-14/dez/1997. Temperatura (T), Potencial Hidrogeniônico (pH), Alcalinidade (A), Salinidade (Sal) e Fosfato total (Ptot).

\begin{tabular}{|c|c|c|c|c|c|c|c|c|c|c|c|c|c|c|c|}
\hline \multirow[b]{2}{*}{ hora } & \multicolumn{3}{|c|}{$\mathrm{T}\left({ }^{\circ} \mathrm{C}\right)$} & \multicolumn{3}{|c|}{$\mathrm{pH}$} & \multicolumn{3}{|c|}{$\mathrm{A}(\mathrm{mEq} / \mathrm{l})$} & \multicolumn{3}{|c|}{$\mathrm{Sal}(\mathrm{mg} / \mathrm{l})$} & \multicolumn{3}{|c|}{ Ptot $(\mu \mathrm{g} / \mathrm{l})$} \\
\hline & I & II & III & I & II & III & I & II & III & I & II & III & I & II & III \\
\hline $17: 30$ & 29 & 24 & 26 & 6,03 & 5,85 & 6,60 & 0,36 & 0,70 & 0,94 & 22 & 60 & 92 & 100 & 120 & 190 \\
\hline $23: 30$ & 24 & 17 & 26 & 4,75 & 6,00 & 6,30 & 0,34 & 0,72 & 0,98 & 22 & 58 & 88 & 100 & 130 & 140 \\
\hline $5: 30$ & 24 & 18 & 26 & 4,60 & 5,75 & 6,30 & 0,38 & 0,72 & 0,94 & 22 & 60 & 92 & 100 & 130 & 210 \\
\hline $11: 30$ & 28 & 27 & 26,5 & 5,41 & 6,13 & 6,30 & 0,38 & 0,72 & 0,90 & 20 & 60 & 76 & 100 & 60 & 220 \\
\hline $17: 30$ & 27 & 27 & 26 & 5,13 & 5,90 & 6,50 & 0,36 & 0,70 & 0,98 & 20 & 58 & 76 & 100 & 120 & 190 \\
\hline
\end{tabular}


Tabela 3. Lista de espécies (Legenda: FB - formas biológicas; Sl- submersa livre; Ff - flutuante fixa; Fl - flutuante livre; E - emergente; A - anfíbia; Ep - epífitos).

\begin{tabular}{llll}
\hline Identificação & FB & Açudes & Testemunho
\end{tabular}

PTERIDOPHYTA identificado por E. Melo

AZOLLACEAE

Azolla filiculoides Lam.

$\begin{array}{lll}\text { Fl } & 1356 & \text { Melo } \text { et al. } 1799 \\ \text { E } & 5 & \text { Melo } \text { et al. } 1737 \\ \text { Fl } & 236 & \text { França } \text { et al. } 1905 \\ \text { Fl } & 5 & \text { Melo } \text { et al. } 1818 \\ \text { E } & 36 & \text { Melo } \text { et al. } 2008\end{array}$

MARSILEACEAE

Marsilea sp.

SALVINIACEAE

Salvinia minima Baker

S. oblongifolia Kuhn.

THELYPTERIDACEAE

Thelypteris interrupta (Willd.) Iwatsuki

MAGNOLIOPHYTA (Magnoliopsida)

ACANTHACEAE identificado por F. França

Ruellia bahiensis (Ness) Morong $\quad$ A 2

$R$. paniculata $\mathrm{L}$.

A $\quad 26$

França et al. 1980

Melo et al. 1762.

AMARANTHACEAE identificado por F. França, M. Bezerra \& L.R. Senna

Alternanthera tenella Colla

Gomphrena demissa Mart.

A $\quad 5$

ASTERACEAE identificado por D.J.N. Hind

Acmella uliginosa (Sw.) Cass.

Ageratum conyzoides L.

A 3

França et al. 1738

França et al. 1994

Blainvillea dichotoma (Murr.) Cass.

A 5

A 5

A 6

Centratherum punctatum Cass.

A 12

Delilia biflora DC.

A 2

Eclipta prostata (L.) L.

A 34

Enydra radicans (Willd.) Lack.

Gamochaeta americana Wedd.

A $\quad 346$

Sonchus oleraceus L.

A 5

E 3

Wedelia alagoensis Baker

A 1

BEGONIACEAE identificado por E.L. Jacques

Begonia fischeri Schrank.

E 6

França et al. 1767

Melo et al. 1722

Melo et al. 1705

França et al. 1983

França et al. 1881

França et al. 1993

França et al. 2029

Melo et al. 1730

França et al. 1987

França et al. 1733

BORAGINACEAE identificado por F. França \& I.S. Freitas

Cordia superba Cham.

Heliotropium cf. angiospermum Murr.

$H$. cf. indicum L.

H. procumbens Mill.

A 5

A 1

A 6

A $\quad 346$

Melo et al. 2009

CAESALPINIACEAE identificado por L.P. de Queiroz

Senna uniflora (P. Miller) Irwin \& Barneby

$\mathrm{A} / \mathrm{E} \quad 125$

França et al. 1978

França et al. 1745

Melo et al. 2012

Melo et al. 2010

CAPPARACEAE identificado por F. França \& L.P. Queiroz

Cleome guianesis Aubl.

A 3

A 6

CONVOLVULACEAE identificado por M.E.R. Junqueira \& R. Simão-Bianchini

Evolvulus filipes Mart.

A 4

A 2

Ipomoea setosa Ker-Gawl.

A $\quad 5$

Merremia umbellata (L.) Hallier f.

A 1

CUCURBITACEAE

Cucumis sp.

A $\quad 26$

Melo et al. 1757

EUPHORBIACEAE identificado por F. França

Chamaesyce hyssopifolia (L.) Small

A $\quad 12$

França et al. 1883 
Tabela 3 (continuação)

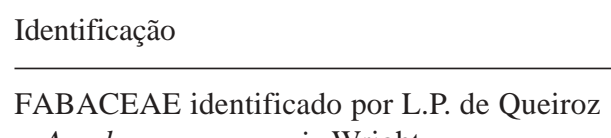

Aeschynomene evenia Wright

Centrosema brasilianum (L.) Benth.

Desmodium tortuosum (Sw.) DC.

Macroptilium lathyroides (L.) Urban

Rhynchosia minima DC.

Stylosanthes guianensis Sw.

HYDROPHYLACEAE identificado por F. França

Hydrolea spinosa $\mathrm{L}$.

LAMIACEAE identificado por F. França

Hyptis suaveolens (L.) Poit.

Marsypianthes chamaedrys (Vahl) O. Kuntze

Ocimum campechianum Mill.

LENTIBULARIACEAE identificado por F. França

Utricularia gibba $\mathrm{L}$.

LOBELIACEAE identificado por F. França

Lobelia xalapense H. B. K.

LYTHRACEAE identificado por S. Graham \& F. França Ammannia latifolia $\mathrm{L}$.

Pleurophora anomala Koehne

MIMOSACEAE identificado por L.P. de Queiroz Mimosa pudica L.

MENYANTHACEAE identificado por F. França

Nymphoides indica $\mathrm{L}$.

NYMPHAEACEAE identificado por G. Pedralli

Nymphaea ampla (Salisb.) DC.

ONAGRACEAE identificado por G. Pedralli

Ludwigia erecta (L.) Hara

L. filiformis T.P. Ram.

L. inclinata (L.f.) Gómez

Ludwigia sp.

MALVACEAE identificado por F. França

Malachra sp.

Sida spinosa L.

PHYTOLACCACEAE identificado por E. Melo

Microtea longebracteata $\mathrm{H}$. Walter

POLYGONACEAE identificado por E. Melo

Polygonum hispidum Kunth

P. ferrugineum Wedd.

PORTULACACEAE identificado por F. França

Portulaca marginata H. B. K.

RUBIACEAE identificado por F. França

Borreria verticillata Griseb.

SCROPHULARIACEAE identificado por F. França

Angelonia salicariaefolia Bonpl.

Bacopa stricta (Schrad.) Wettst. ex Edwall

Melasma melampyroides (Rich.) Pennell

Micranthemum umbrosum (Walter ex Gmel.) Blake

Scoparia dulcis L.

Stemodia pratensis (Aubl.) C.P. Cowan
FB

Açudes

Testemunho

A

A

A

A

A

A

A

A

A

S1

A

$\mathrm{A} / \mathrm{E}$

$\mathrm{A} / \mathrm{E}$

1456

12345

A

25

Ff

2

Ff

123456

Melo et al. 1819

$\mathrm{A} / \mathrm{E} \quad 6$

A 26

A/Ff 245

$\mathrm{A} / \mathrm{E}$

3

A 2

A 5

A 3

$\mathrm{A} / \mathrm{E} \quad 56$

A/E 234

A 3

A $\quad 15$

Melo et al. 1735

Melo et al. 1721

França et al. 2007

França et al. 1880

Melo et al. 1733

França et al. 1895

Melo et al. 1725 
Tabela 3 (continuação)

Identificação $\quad$ FB Açudes Testemunho

SOLANACEAE identificado por E. Melo \& F. França

Physalis pubescens L.

Solanum paniculatum $\mathrm{L}$.

TILIACEAE identificado por F. França

Corchorus hirtus L.

TURNERACEAE identificado por F. França

Piriqueta racemosa (Jacq.) Sweet

Turnera sp.

VERBENACEAE identificado por F. França \& S. Atkins

Priva bahiensis DC.

Stachytarpheta elatior Schrad.

MAGNOLIOPHYTA (Liliopsida)

ARACEAE identificado por F. França

Pistia stratiotes L.

ALISMATACEAE identificado por A. Oliveira

Echinodorus grandiflorus Mitch.

E. subalatus (Mart.) Griseb.

COMMELINACEAE identificado por F. França \& A.M. Giulietti

Callisia filiformis (Mart. \& Galeotti.) D.H. Hunt

Commelina cf. erecta $\mathrm{L}$.

CYPERACEAE identificado por G. Pedralli, M. Alves, E.A. Rocha \& A. Araújo

Cyperus aggregatus (Willd.) Endl.

C. cayannensis (Lam.) Britton

C. cf. hermafroditus (Jacq.) Stand.

C. esculentus L.

C. ferax Rich.

C. haspan $\mathrm{L}$.

C. iria L.

C. lanceolatus Poir.

C. odoratus $\mathrm{L}$.

C. polystachyus Rottb.

Cyperus sp.

C. surinamensis Rottb.

C. virens Michx.

Eleocharis nodulosa Schult.

Fimbristylis diphylla (Reitz) Vahl

Oxycaryum cubense (Poepp. \& Kunth) Lye

Pycreus macrostachyos (Lam.) Raynal

Rhynchospora contracta (Ness) J. Raynal.

HYDROCHARITACEAE identificado por F. França

Limnobium laevigatum Humb. \& Bonp. ex Willd.

LEMNACEAE identificado por M. Bezerra

Lemna aequinoctialis Welw.

L. cf. valdiviana Pohl

Wolffia brasiliensis Wedd.

Wolffiella welwitschii (Hegelm.) Monod.

LIMNOCHARITACEAE identificado por F. França

Hydrocleys nymphoides Buch.

NAJADACEAE identificado por E. Melo

Najas conferta A. Br.
A $6 \quad$ França et al. 1648

A $1 \quad$ França et al. 1875

A $\quad 26 \quad$ França et al. 1756

A 2 França et al. 1989

A 2 França et al. 1982

A $2 \quad$ França et al. 1884

A $\quad 1235 \quad$ França et al. 1998

Fl $\quad 123456$ França et al. 2033

E $\quad 256 \quad$ Melo et al. 1698

E 4 França et al. 1640

A $245 \quad$ Melo et al. 1732

A/E $\quad 256 \quad$ França et al. 2012

$\begin{array}{lll}\text { A } & 2 & \text { França } \text { et al. } 2211\end{array}$

A/E $\quad 1 \quad$ Melo et al. 1988

A/E $\quad 5 \quad$ Melo et al. 1726

A $\quad 16 \quad$ França et al. 1732

A/E $\quad 1256 \quad$ Melo et al. 1994

A/E $235 \quad$ Melo et al. 2000

E $4 \quad$ Melo et al. 1746

E 3 França et al. 2225

E 55 França et al. 2236

A/E $4 \quad$ França et al. 2004

A $3 \quad$ França et al. 2217

A/E $\quad 12456 \quad$ Melo et al. 1723

A/E $\quad 16 \quad$ França et al. 1736

A/E $\quad 456 \quad$ França et al. 1769

E $\quad 35 \quad$ França et al. 2241

Ep/E $\quad 123456 \quad$ Melo et al. 1821

E $3 \quad$ França et al. 2228

A/E $\quad 135 \quad$ Melo et al. 1731

Fl $\quad 235 \quad$ Melo et al. 1754

Fl $\quad 1356 \quad$ Melo et al. 1657

Fl $\quad 123456 \quad$ Melo et al. 1766A

Fl $\quad 1346 \quad$ Melo et al. 1662

Fl $\quad 13456 \quad$ Melo et al. 1658B

Ff $\quad 235 \quad$ França et al. 1899

S1 $\quad 6 \quad$ Melo et al. 2015

continua 
Tabela 3 (continuação)

\begin{tabular}{|c|c|c|c|}
\hline Identificação & FB & Açudes & Testemunho \\
\hline \multicolumn{4}{|c|}{ ORCHIDACEAE identificado por A. Toscano de Britto } \\
\hline Habenaria repens Nutt. & $\mathrm{E}$ & 356 & França et al. 2359 \\
\hline \multicolumn{4}{|c|}{ POACEAE identificado por H. Wagner \& R.P. Oliveira } \\
\hline Anthephora hermaphrodita (L.) O. Kuntze & A & 3 & França et al. 1990 \\
\hline Chloris barbata $\mathrm{Sw}$. & $\mathrm{A} / \mathrm{E}$ & 15 & França et al. 1878 \\
\hline C. exilis Renvoize & A & 2 & França et al. 1991 \\
\hline Echinochloa colona (L.) Link & $\mathrm{A} / \mathrm{E}$ & 123456 & França et al. 2207 \\
\hline Eragrostis pilosa (L.) P. Beauv. & A & 34 & França et al. 1892 \\
\hline Hymenachne amplexicaulis (Rud.) Nees & $\mathrm{A} / \mathrm{E}$ & 23 & França et al. 1986 \\
\hline Leptochloa aff. fascicularis (Lam.) A. Gray & $\mathrm{E}$ & 3 & França et al. 2216 \\
\hline Luziola brasiliana Moric. & $\mathrm{E}$ & 3 & Melo et al. 2002 \\
\hline Panicum boliviensis Hackel & A & 1246 & França et al. 2032 \\
\hline P. maximum Jacq. & $\mathrm{A} / \mathrm{E}$ & 1 & França et al. 2205 \\
\hline Panicum sp. 1 & A & 4 & França et al. 2231 \\
\hline Panicum sp. 2 & A & 6 & Melo et al. 1709 \\
\hline Paspalidium geminatum (Forsk.) Stapf & $\mathrm{A} / \mathrm{E}$ & 6 & Melo et al. 1807 \\
\hline \multicolumn{4}{|l|}{ PONTEDERIACEAE identificado por M. Bezerra } \\
\hline Eichhornia crassipes (Mart.) Solms & $\mathrm{Fl} / \mathrm{E}$ & 456 & Melo et al. 1805 \\
\hline E. paniculata (Spreng) Solms & $\mathrm{A} / \mathrm{E}$ & 256 & França et al. 1770 \\
\hline \multicolumn{4}{|l|}{ TYPHACEAE identificado por F. França } \\
\hline Typha domingensis Pers. & $\mathrm{E}$ & 356 & França et al. 1889 \\
\hline
\end{tabular}

Quanto ao tipo de forma biológica, a maioria das espécies amostradas são Anfíbias (73\%), seguido pelas Emergentes (35\%), Anfíbias e Emergentes (17\%), Flutuantes Livres (8\%) e Flutuantes Fixas (3\%). Apenas Oxycarium cubense pode ser identificada como Epífita, pois foi encontrada nesta forma em todos os açudes estudados.

\section{Discussão}

Apesar da maioria das espécies encontradas enquadrarem-se nas Anfíbias, grande parte dessas correspondem a espécies ruderais, comuns nos pastos da região, que ocorrem apenas ocasionalmente em ambiente aquático. As espécies efetivamente aquáticas ou de ambientes brejosos são as Emergentes, Anfíbias e Emergentes, Flutuantes Livres, Flutuantes Fixas e epífitas.

Os índices de similaridade de Sørensen (S) observados são considerados baixos, de acordo com a regra geral para S (Felfili et al. 1994).
Observando os dados de espécies comuns entre dois açudes, verifica-se que a distância geográfica entre os açudes não influenciou no compartilhamento, pois os açudes 2 e 5, que apresentaram grande número de espécies em comum e aparecem formando grupos similares em todas as análises de agrupamento, além de estarem distantes (ca. 13km), não pertencem ao mesmo sistema de micro-bacias.

Paralelamente, os açudes 1 e 2, que são vizinhos, não formam um grupo em nenhum dendrograma, ao contrário, os dendrogramas mostram que o açude 1 parece ser mais relacionado com os açudes 4 e 6 . Esse resultado, associado ao fato de que o açude 1 apresenta alta alcalinidade em relação ao açude 2 , não corrobora a idéia de que as águas dos dois açudes se comuniquem por baixo da estrada, aparentando serem dois açudes com origens diferentes.

As concentrações de fósforo total corresponderam às maiores concentrações observadas por Marinho (1994), ao analisar as águas do 
fundo de um açude em Mogi Guaçu (São Paulo).

A leve acidez observada na água dos açudes estudados pode estar relacionada com a presença de grandes populações de macrófitas, o que também foi observado por Marinho (1994). Corrobora-se esta afirmação com a observação de que em águas pouco povoadas de macrófitas o pH médio mostra-se acima de 7 (Moura 1996; Ramirez 1996).

Os menores açudes ( 1 e 4 ) foram aqueles que apresentaram o menor número de espécies exclusivas e foram também os que apresentaram maiores discrepâncias em relação a alguns parâmetros físico-químicos, o maior valor de alcalinidade (açude 1) e salinidade (açude 4).

Considerando a temperatura do ar, a área de estudo pode ser considerada quente, comparável aos setores mais quentes do nordeste brasileiro. Isso associado ao fato que $58 \%$ do período constituiu-se de meses secos, pode-se considerar o clima da área de estudos como semi-árido moderado, de acordo com a classificação climática exposta em Nimer (1989).

As coletas de água durante $24 \mathrm{~h}$ mostrararam que os valores variam pouco durante o dia, mas também mostraram melhor as relações destes valores com as variações climáticas sazonais. A temperatura é significativamente mais baixa nos períodos mais úmidos. $\mathrm{O} \mathrm{pH}$ é significativamente mais ácido nos períodos úmidos, resultado este que corrobora a idéia de que o grande desenvolvimento de macrófitas está relacionado com o aumento da acidez da água, o que ocorreu neste período. A alcalinidade foi mais baixa no período úmido, corroborando os dados de $\mathrm{pH}$. A salinidade foi mais baixa no período mais úmido e mais alta no período mais seco. O mesmo foi observado com os valores de fosfato total, sendo estes significativamente mais altos nos períodos mais secos.

$\mathrm{O}$ açude 2 mostrou a menor salinidade. Note-se que Wolffiella welwitschii, com ocorrência em todos os outros açudes, não foi observada neste. Além disso, esta espécie é muito freqüente no açude 1. Estes resultados sugerem uma influência da baixa salinidade na ocorrência daquela espécie.

A ocorrência de Utricularia gibba também sugere influência dos fatores físico-químicos, uma vez que esta espécie só não foi encontrada na açude 1 , justamente o açude mais alcalino e ocorrendo no vizinho açude 2 .

Mais estudos devem ser feitos para melhor demonstrar os efeitos da alcalinidade nas ocorrência de Utricularia gibba e da salinidade em Wolffiella welwitschii.

\section{Agradecimentos}

Os autores agradecem ao sr. Pedro Manuel de Oliveira, que permitiu o desenvolvimento deste trabalho em sua propriedade (Fazenda Caraíbas: açude 5); ao biólogo Roy Funch, da Fundação Chapada Diamantina, pela revisão do Abstract.

\section{Referências bibliográficas}

Albuquerque, B. W. P. 1981. Plantas forrageiras da Amazônia. I-Aquáticas Flutuantes, Livres. Acta Amazônica 11(3): 457-472.

Bahia. 1995. Municípios da região semi-árida. Escala 1:2.000.000. Superintendência de Estudos Econômicosda Bahia (SEI), Governo do Estado da Bahia, Salvador.

Brasil. s.d. Folha topográfica SD 24-V-B. Escala 1: 250.000. Instituto Brasileiro de Geografia e Estatística, Salvador.

Clesceri, L. S.; Greenberg, A. E. \& Trussel, R. R. 1989. Standard methods for the examination of water and wastewaters. $17^{\text {th }} \mathrm{ed}$. APHA/AWWA/WPCF, Washington.

Crisci, J. V. \& Armengol, M. F. 1983. Introduccion a la teoria y practica de la taxonomia numerica. Organización de los Estados Americanos, Washington.

Cronquist, A. 1981. An integrated system of classification of flowering plants. Columbia University Press, New York.

Felfili, J. M.; Haridassan, M.; Mendonça, R. C.; Filgueiras, T. S.; Silva Junior, M. C. \& Rezende, A. V. 1994. Projeto biogeografia do bioma cerrado: vegetação \& solos. Cadernos de Geociências 12: 75-166. 
Fidalgo, O. \& Bononi, V. R. L. 1984. Técnicas de coleta, preservação e herborização de material botânico. Instituto de Botânica, São Paulo.

Irgang, B.; Pedralli, G. \& Waechter, J. L. 1984. Macrófitos aquáticos da Estação Ecológica do Taim, Rio Grande do Sul, Brasil. Roessléria 6(1): 395-404.

Marinho, M. M. 1994. Dinâmica da comunidade fitoplanctônica de um pequeno reservatório raso, densamente colonizado por macrófitas aquáticas submersas (Açude do Jacaré, Mogi Guaçu, SP, Brasil). Dissertação de Mestrado. Universidade de São Paulo, São Paulo.

Moura, A. 1996. Estrutura da comunidade fitoplanctônica numa lagoa eutrófica, São Paulo, SP, Brasil, a curtos intervalos de tempo: comparação entre as épocas de chuva e seca. Dissertação de Mestrado. Universidade de São Paulo, São Paulo.

Mueller-Dumbois, D. \& Ellenberg, H. 1974. Aims and methods of vegetation ecology. John Wiley \& Sons, New York.
Nimer, E. 1989. Climatologia do Brasil. $2^{\mathrm{a}}$ ed. IBGE, Rio de Janeiro.

Pedralli, G. 1996. Aguapés: Biologia, manejo e uso sustentado. Estudos de biologia - PUCPR 4(40): 33-53.

Ramirez R., J. J. 1996. Variação espacial, vertical e nictemeral da estrutura da comunidade fitoplanctônica e variáveis ambientais em quatro amostragens em diferentes épocas do ano no Lago das Garças, São Paulo. Tese de Doutorado. Universidade de São Paulo, São Paulo.

Rohlf, F. J. 1997. NTSYS-PC: numerical taxonomy and multivariate analyses system. Exeter Software, New York.

Tryon, R. M. \& Tryon, A. F. 1982. Ferns and allied plants with special reference to tropical America. Springer-Verlag, New York.

Tundisi, J. G. 1994. Tropical South America: present and perspectives. Pp. 353-424. In: R. Margalef (ed.). Limnology now: a paradigm of planetary problems. Elsevier Science B.V., Amsterdam. 